\title{
SQLE inhibition suppresses the development of pancreatic ductal adenocarcinoma and enhances its sensitivity to chemotherapeutic agents in vitro
}

Fengyun Zhao ( 20193101013@stu.gzucm.edu.cn )

Guangzhou University of Chinese Medicine https://orcid.org/0000-0003-2266-066X

\section{Yanni Huang}

Guangzhou University of Chinese Medicine

\section{Yinheng Zhang}

Guangzhou University of Chinese Medicine

\section{Xun Li}

South China Normal University

\section{Caizhao Chen}

South China Normal University

\section{Yihao Long}

South China Normal University

\section{Fugui Li}

Zhongshan People's Hospital

\section{Xiaodong Ma}

South China Normal University

\section{Research Article}

Keywords: Pancreatic ductal adenocarcinoma (PDAC), Squalene epoxidase (SQLE), Cholesterol biosynthesis, mTORC1, NF-KB

Posted Date: February 15th, 2022

DOI: https://doi.org/10.21203/rs.3.rs-1330088/v1

License: (c) (i) This work is licensed under a Creative Commons Attribution 4.0 International License. Read Full License 


\section{Abstract}

\section{Purpose}

In this study, we sought to explore the function of seven important enzymes(MSM01, EBP, HMGCS1, IDI2, DHCR7, FDFT1, and SQLE) involved in cholesterol biosynthesis especially SQLE in PDAC therapy.

\section{Methods and results}

The TCGA and Oncomine dataset were used to explore the expression of the seven enzymes in normal and cancerous pancreatic individual, and their anti-proliferation efficiency against PDAC cells was measured by cell viability assay. Expression level and prognostic values of SQLE were evaluated by western blot and Kaplan-Meier analysis. The influence of SQLE knockdown by shRNA in PDAC cells was assessed by Transwell, colony formation and cell cycle analysis. RNA-seq and GSEA were utilized to investigate the potential mechanisms. The synergistic effect of SQLE inhibitor, terbinafine, combined with six chemotherapeutic drugs in PDAC cells was tested by CCK-8 method. We demonstrated that downregulation of those enzymes especially SQLE significantly suppressed PDAC cells survival. SQLE was upregulated in PDAC cell lines, and the elevated level of SQLE is correlated with poor prognosis in pancreatic cancer samples. SQLE knockdown could significantly inhibit the proliferation and migration of PDAC cells. Cell cycle was blocked in S phase after SQLE silencing. Mechanistically, GSEA analysis with RNA-seq data revealed that SQLE silencing negatively mediated mTORC1 and TNFa/NF-KB signaling pathways. Besides, SQLE inhibitor terbinafine enhanced chemotherapeutic sensitivity of the six compounds.

\section{Conclusions}

This study demonstrated that SQLE is a novel target for PDAC therapy. The synergism role of SQLE inhibition and chemotherapy may be potential therapeutic strategies for pancreatic cancer treatment.

\section{Introduction}

Pancreatic ductal adenocarcinoma (PDAC) represents one of the most aggressive malignant diseases with an extremely high morbidity and mortality [1]. Surgery is the only potential curative treatment for PDAC. However, most PDAC patients are diagnosed at advanced disease stages with no opportunity for radical surgery. For advanced PDAC patients, chemotherapy is the main strategy for disease control. Despite multiple chemotherapy regimens have been applied to improve treatment outcomes over the last several decades, prognosis of PDCA remains poor, with a dismal overall 5-year survival rate of $5 \%$ [2]. Previous studies have explored therapies targeting KRAS, MAP2K4, TP53, CDKN2A, SMAD4 and TGF $\beta 22$ mutated genes, which are recognized as major drivers of pancreatic carcinogenesis, while the results demonstrated no meaningful survival benefit in PDAC [3]. Although immune checkpoints blockade (ICB) therapy with PD-1 and PD-L1 antibodies has shown durable response rate in multiple immunogenic tumors, PDAC is not included. Hence, identification of new strategies for PDAC is urgently needed. 
Recent advances in cancer metabolism have revealed that many abnormal metabolism patterns are potential targets for cancer treatment. Among various metabolic pathways, cholesterol metabolism alterations have been shown to be related to tumorigenic processes of PDAC [4]. Cholesterol is an essential substance for cell proliferation, differentiation and membrane formation, which is involved in tumor proliferation and death. In this process, cancer cells synthesize a large amount of cholesterol to meet the energetic demands associated with rapid proliferation. There are many enzymatic reactions in cholesterol metabolism. Among them, SQLE is one of the rate-limiting enzymes in the downstream of cholesterol synthesis, which catalyzes the synthesis of sterol and cholesterol from 2,3 (s) squalene. Previous study showed that lipid metabolism was disturbed in the brain and liver of male mice with MeCP2 deficiency, and cholesterol homeostasis was considered as a potential target for Rett syndrome treatment [5]. And SQLE is highly expressed in a variety of malignant tumors, such as breast cancer [6] primary lung cancer $[7,8]$, prostate cancer [9], and liver cancer $[10,11]$. The overexpression of SQLE can be used as a predictor of poor prognosis in breast cancer [6]. Highly expressed SQLE can inhibit the expression of PTEN and promote Akt-mTOR pathway in hepatocellular carcinoma (HCC) caused by nonalcoholic fatty liver, which is considered as an effective potential target for HCC [11]. Abnormal expression of SQLE is also proved to be a potential biomarker and therapeutic target for the treatment and prognosis of lung squamous cell carcinoma [8]. However, the specific function and mechanism of SQLE in pancreatic cancer has not been elucidated.

In this study, using loss-of-function method, we evaluated the anti-proliferation efficiency against PDAC cells of seven important enzymes involved in cholesterol metabolism, SQLE displayed significant inhibition effect on the proliferation of all the tested cell lines. The expression of SQLE was upregulated in PDAC cell lines, and the elevated SQLE levels correlated with poor prognosis, indicating SQLE was closely related to the development of pancreatic cancer. More importantly, SQLE knockdown could significantly inhibit the proliferation and migration of PDAC cell line PANC-1. Cell cycle of PANC-1 cells was blocked in $S$ phase after SQLE silencing. Mechanistically, GSEA analysis with RNA-seq data revealed that SQLE silencing negatively mediated mTORC1 and TNFa/NF-KB signaling pathways. Besides, the synergistic inhibition effect of terbinafine, a SQLE inhibitor, combined with six chemotherapeutic drugs (cisplatin, gemcitabine, 5-Fluorouracil, THZ1, (+)-JQ-1 and panobinostat) on the proliferation of PDAC cells was verified. Terbinafine enhanced chemotherapeutic sensitivity of all the tested compounds, especially with panobinostat. In conclusion, these findings identified SQLE as a new target for anticancer therapy in PDAC, and modulating SQLE with chemotherapeutic agents may be a potential therapeutic strategy for pancreatic cancer treatment.

\section{Materials And Methods}

\section{Cell culture and reagents}

Human normal pancreatic cell line (H6C7) and PDAC cell lines (PANC-1, CFPAC1, BXPC3, PACA2, SW1990) were purchased from the Cell Bank of the Chinese Academy of Sciences (Shanghai, China) and cultured in Dulbecco's modified Eagle's medium (DMEM, Gibco) supplemented with 10\% FBS and 1\% 
penicillin/streptomycin. All cells were incubated at $37^{\circ} \mathrm{C}$ in humidified air containing $5 \% \mathrm{CO} 2$.

Panobinostat(T2383), THZ1(T3664), Gemcitabine(T0251), (+)-JQ-1(T2110), cisplatin(T1564), 5Fluorouracil(T0984) and terbinafine(T6702) were purchased from Target Mol (Shanghai, China).

\section{Cell viability assay}

Cell counting kit-8 (CCK-8, Dojindo) assay was applied to detect cell proliferation. Cells were inoculated in a 96-well plate at a density of $3 \times 103$ cells/well. After $48 \mathrm{~h}$ incubation with different treatments, $10 \mu \mathrm{L}$ CCK8 reagent was added to each well and incubated at $37^{\circ} \mathrm{C}$ for another $3 \mathrm{~h}$. Subsequently, the absorbance at $450 \mathrm{~nm}$ of each well was determined by microplate reader. The wells were evaluated in triplicate and the experiment were repeated three times.

\section{Colony formation assay}

Cells were seeded in 6 -well plates at a density of 500 cells/well and incubated at $37^{\circ} \mathrm{C}$. Cell culture medium were refreshed every 3 days until colonies formed. Following cell fixation with paraformaldehyde solution, crystal violet was utilized to stain the cells. Colonies with more than 50 cells per colony were counted under a microscope.

\section{Transwell assay}

The invasive abilities of cancer cells were tested using a 24-well transwell plate with $8 \mu \mathrm{m}$ pore polycarbonate membrane inserts (Corning). Cells $(2 \times 104)$ in $200 \mu \mathrm{L}$ serum-free medium were seeded in the upper chamber and the lower chamber was loaded with $600 \mu \mathrm{L}$ complete medium. After cultured at $37^{\circ} \mathrm{C}$ for $24 \mathrm{~h}$, cells migrated to the lower membrane surface were fixed with $4 \%$ formaldehyde and stained with crystal violet. The migration of PANC-1 cells was then detected under a microscope.

\section{Cell cycle analysis}

Cells were seeded in a 6-well plate at a density of $1 \times 106$ cells/well. After different treatments, cells were harvested and fixed with $70 \%$ ethanol overnight at $-20^{\circ} \mathrm{C}$. Subsequently, the fixed cells were washed twice with PBS and stained with propidium iodide (PI; BD, USA) and RNase for $25 \mathrm{~min}$. Cell cycle was analyzed by flow cytometry (Accuri C6, BD Biosciences).

\section{Plasmid construction and transfection}

PANC-1, BXPC3, and CFPAC1 cells were transfected with control shRNA(shControl), SQLE shRNA(shSQLE), FDFT1 shRNA(shFDFT1), HMGCS1 shRNA(shHMGCS1), DHCR7 shRNA(shDHCR7), EBP shRNA(shEBP), MSM01 shRNA(shMSM01), and IDI2 shRNA(shIDI2) by using Lipofectamine RNAi MAX reagent. Briefly, 293T cells were seeded in a 6-well plate and transfected with PRSV-rev $(0.75 \mu \mathrm{g})$, PMDLrre $(0.75 \mu \mathrm{g})$, PMD2.G $(0.75 \mu \mathrm{g})$, and shRNA plasmid $(2 \mu \mathrm{g})$ per well. After $48 \mathrm{~h}$ incubation, virus supernatant was collected by centrifugation at $2000 \mathrm{rpm}$ for $10 \mathrm{~min}$ followed by filtration through 0.45 $\mu \mathrm{m}$ filter. The PDAC cell lines were infected with $500 \mu \mathrm{L}$ virus supernatant containing $8 \mu \mathrm{g} / \mathrm{mL}$ of polybrene for $6 \mathrm{~h}$. Culture medium was refreshed for another $48 \mathrm{~h}$ of incubation, and cells were then collected for western blot analysis. The shRNA sequences for genes were as follows: shControl: 5'- 
GGTGTGCAGTTGGAATGTA-3'; shSQLE: 5'-GCTCAGGCTCTTTATGAATTA-3'; shHMGCS1: 5'CCCATCATTTGGTCAACTATA-3'; sh FDFT1: 5'- GTGTTTAACTTCTGTGCTATT-3'; shDHCR7: 5'CCCTGACTTCTGCCATAAGTT -3'; shEBP: 5'-GCACCTAAGACTGGACAACTT-3'; shMSM01: 5'TGATTTGTGGAACCTATTATT-3'; shIDI2: 5'- CCTTCAGCGTTGTCTTGTTTA-3'.

\section{Western blot}

Total protein of cells was extracted using RIPA lysis buffer. Protein concentrations were determined using a BCA protein assay kit (Beyotime Biotechnology). SDS-PAGE electrophoresis was used to sperate proteins. Then, the separated proteins were transferred onto the PVDF membrane, and blocked with $5 \%$ skim milk for $1 \mathrm{~h}$. After that, PVDF membrane was incubated with primary antibody GAPDH(Abcam) (1:10000) and SQLE (Proteintech) (1:2000) at $4^{\circ} \mathrm{C}$ overnight. Subsequently, the membrane was incubated with HRP-conjugated anti-rabbit or murine secondary antibody (Abcam) (1:10000) for $1 \mathrm{~h}$ at room temperature after being washed three times with TBST. Finally, the immunoreactive proteins were visualized with SuperSignal West Dura Chemiluminescent (Thermo Fisher).

\section{RNA-sequencing and bioinformatics analysis}

Total RNA was extracted for RNA-sequencing (RNA-Seq) by using the TRI Reagent and Direct-zol RNA kit (Zymo Research, Irvine, CA, USA) following the manufacturer's instructions. The sequencing was performed by a sequencing service company (Novogene, Beijing, China) using the Illumina sequencing platform. Log2|Fold change| $\geq 1$ and $P<0.05$ were used as a cut-off to define overexpression or downregulation. Gene Set Enrichment Analysis (GSEA) was then performed to reveal the signaling pathways in which differentially expressed genes were enriched.

\section{Statistics analysis}

Data are presented as mean \pm SD. Data were analyzed via the Student's t test using GraphPad Prism 8.0 software. The statistical difference $P<0.05$ was considered to be significant.

\section{Results}

\section{The role of cholesterol metabolism associated genes in cell proliferation of PDAC}

Cholesterol synthesis is a complex biological process includes multiple enzymes encoded by MSM01, EBP, HMGCS1, IDI2, DHCR7, FDFT1, and SQLE (Fig. 1A). To explore their roles in pancreatic cancer, we first analyzed their expression in PDAC by using ONCOMINE and TCGA database. As shown in Fig. 1B and Fig. 1C, only SQLE and MSM01 were significantly overexpressed in pancreatic tumor tissues compared to their adjacent tissues or normal tissues. To further investigate the function of these 7 genes in multiple PDAC cell lines including PANC-1, CFPAC1 and BXPC3, we constructed shRNA to knockdown these genes in cell lines, and evaluated cell proliferation at 24, 48 and $72 \mathrm{~h}$ after gene silencing. Our results showed that knocking down these genes in three PDAC cell lines significantly suppressed cell 
proliferation(Fig. 1D-F). In particular, knockdown of SQLE displayed most significant inhibition effect on the proliferation than other 6 genes. Thus, we focused on SQLE in the further experiments.

\section{SQLE is up-regulated and promotes tumor progression in PDAC}

To explore the expression signature of SQLE, we first evaluated the transcription level of SQLE in human normal tissues from GTEx. And the data showed that SQLE level was highly abounded in esophagus, brain and skin tissue, while it represents a relative low expression in muscle, heart and pancreatic tissues (Fig. 2A). Next, we evaluated the expression of SQLE in multiple solid tumors based on the TCGA database, and the result showed that SQLE was overexpressed in most solid tumors including PDAC, which indicates the critical role of SQLE in tumorigenesis(Fig. 2B). To confirm these findings, the expression of SQLE in six pancreatic cancer cell lines, PANC-1, BXPC3, CFPAC1, ASPC1, SW1990 and PACA2 was measured by western blot, with pancreatic duct epithelial cell ( $\mathrm{H6C7})$ as control group. The results indicated that SQLE expression level was significantly increased in pancreatic cancer cell lines compared with H6C7 (Fig. 2C). Moreover, PDAC patients with higher SQLE expression had shorter OS than that with lower SQLE expression (Fig. 2D). To explore the potential role of SQLE in cell proliferation and migration of PDAC, lentivirus-delivered SQLE shRNA vector(shSQLE) and control shRNA vector(shControl) were constructed and were transfected into PANC-1 and CFPAC1 cells. As shown in Fig. 2E, shSQLE remarkably decreased the expression of SQLE in both PANC-1 and CFPAC1 cell lines. The colony formation assays showed that silencing SQLE in PANC-1 and CFPAC1 cells significantly reduced the number of colonies (Fig. 2F). Next, we measured the cell migration of PANC-1 via transwell assay. Knockdown of SQLE inhibited the cellular migration of PANC-1 cells (Fig. 2G). Moreover, PANC-1 cells steady transfected with shSQLE showed an increase in S phase cell population and a decrease in G1 phase cell population (Fig. 2H). Taken together, these observations indicated up-regulated SQLE expression promoted tumor progression in PDAC and was associated with poor prognosis in patients.

\section{SQLE silencing negatively modulates MTORC1 and TNFa/NF-KB signaling in pancreatic cancer cells}

To explore the mechanism underlying SQLE silencing in PDAC cell proliferation, we performed RNAsequencing (RNA-seq) on PANC-1 cells transfected with shControl or shSQLE. A total of 1023 differently expressed genes (DEGs) were identified (Fig. 3A). Gene Set Enrichment Analysis (GSEA) revealed the most enriched canonical pathways involved in the mTORC1 signaling and TNFa/NF-KB signaling (Fig. 3B-3D). These findings indicated that SQLE silencing negatively mediated mTORC1 and TNFa/NF$\mathrm{KB}$ signaling pathway to suppress pancreatic cancer cells proliferation.

\section{SQLE inhibition enhances chemotherapeutic sensitivity in PDAC cell lines}


Given the important oncogenic role of SQLE in PDAC, we evaluated whether SQLE silencing have synergistic effect on the inhibition of PDAC cells proliferation in combined with Cchemotherapeutic agents. Six chemotherapeutic compounds including cisplatin, gemcitabine, 5-Fluorouracil(5FU), THZ1, (+)-JQ-1(JQ1) and panobinostat were used. The results showed that knockdown of SQLE by shSQLE significantly enhanced the inhibition effect of PANC-1 cells proliferation induced by chemotherapeutic compounds (Fig. 4A). Terbinafine is an FDA-approved antifungal drug, which targets SQLE. We next evaluated the viability of PANC-1 cells treated with terbinafine combined with different doses of other six chemotherapeutic compounds. The combined therapy of terbinafine with those chemotherapeutic drugs markedly enhanced the inhibition of cell multiplication compared with therapies using either of these drugs alone, and the results indicated terbinafine had a strongest synergistic interaction with panobinostat (Fig. 4B). To further validate the effective synergism role of terbinafine and panobinostat in PDAC, we further tested the synergistic effect of terbinafine and panobinostat combined therapy in other PDAC cell lines (PACA2, SW1990, CFPAC1, BXPC3, ASPC1). The results showed that the combination of terbinafine and panobinostat had synergistic effects on the inhibition of cell multiplication in all of these PDAC cell lines (Fig. 4C). Collectively, these results suggested that the combination of SQLE inhibitor with chemotherapeutic compounds could be a potential therapeutic strategy for PDAC treatment.

\section{Discussion}

Pancreatic cancer especially PDAC is a fatal malignant disease with extremely low life expectancy. There are no effective options for PDAC treatment besides surgical therapy. But PDAC patients usually have no chance to surgery, which results in rather low OS rate and poor prognosis. Therefore, identifying novel therapeutic targets are potential strategies for improving survival rate of PDAC patients.

In the past several years, many studies have recognized that cholesterol metabolism played a critical role in the development of PDAC $[12,13]$. Cholesterol is not only the essential constituent for maintaining cell homeostasis, but also an important component of plasma membrane [14]. Among numerous enzymes regulating the complex process of cholesterol synthesis, SQLE is one of the most important rate-limiting enzymes [15]. SQLE participates in catalyzing the second rate-controlling step in cholesterol biosynthesis and metabolism [16]. Increasingly studies have reported that dysregulation of SQLE was observed in different malignant tumors, including breast cancer [6], hepatocellular carcinoma [10], squamous cell carcinoma [7] and prostate cancer [9]. Thus, SQLE is an important target in a variety of tumors.

In this study, we identified SQLE as a novel target in PDAC treatment. We found that compared with other genes that modulating cholesterol metabolism, SQLE was remarkably overexpressed in pancreatic cancer cell lines. To evaluate the clinical significance of SQLE in PDAC, we performed the OS analysis based on SQLE expression level. The result revealed that high SQLE expression was correlated with poor prognosis in PDAC. Moreover, SQLE silencing in PANC-1 cells significantly inhibited cell proliferation and migration, blocked the cell cycle in S phase. These findings indicated that SQLE was a potential target for PDAC therapy. 
Previous studies have shown that overexpressed SQLE in HCC inhibited the expression of PTEN, leading to the enhancement of tumor cell proliferation and promotion of tumor development [10]. Abnormal expression of SQLE in primary invasive breast cancer is related to the activation of MYC [5] and cancerassociated microprotein CASIM01, which interacts with SQLE in modulating lipid droplet formation [17]. SQLE has also been reported to promote the development of lung squamous cell carcinoma by activating ERK signaling pathway [7]. In addition, it was revealed that SQLE could induce epithelial to stromal transformation by regulating miR-133b in esophageal squamous cell carcinoma [18]. In present study, we silenced SQLE expression in PANC-1 cells and RNA-seq data analysis by GSEA demonstrated that SQLE silencing could negatively regulate mTORC1 and TNFa/NF-KB signaling pathway. Besides, knockdown of SQLE could enhance the sensitivity of PANC-1 cells to chemotherapeutic drugs. As expected, the combination of SQLE inhibitor terbinafine and chemotherapeutic drugs improved the curative effect and achieve a synergistic effect, and the combination of terbinafine and panobinostat had strongest synergistic effects in all of the tested PDAC cell lines, which provided a potential treatment strategy for PDAC. However, there are still some limitations in this study. Firstly, the effect of SQLE knockdown on PDAC tumor proliferation in vivo required to be verified in the future. Secondly, the mechanism of SQLE inhibition negatively regulates $\mathrm{mTORC1}$ and TNFa/NF-KB signaling pathway is also need to be further investigated.

\section{Conclusions}

In summary, this study demonstrates that SQLE is a novel target for PDAC therapy. The synergism role of SQLE inhibition and chemotherapy may be potential therapeutic strategies for pancreatic cancer treatment.

\section{Declarations}

\section{Data Availability}

The RNA-seq data that support the findings of this study are openly available in figshare at http://doi.org/10.6084/m9.figshare.16740286.

\section{Acknowledgements}

\section{Funding:}

This work was supported by National Natural Science Foundation of China $(81772533,81773249$, 82072697) and the Public Welfare Scientific Research Project of Zhongshan City (No. 2019B1015).

\section{Author information}


Fengyun Zhao, Yanni Huang and Yingheng Zhang have contributed equally to this work.

\section{Affiliations}

The Research Center of Basic Integrative Medicine, Guangzhou University of Chinese Medicine, Guangzhou, 510006, Guangdong, China.

Fengyun Zhao, Yanni Huang \& Yingheng Zhang

Institute for Brain Research and Rehabilitation, Guangdong Key Laboratory of Mental Health and Cognitive Science, Center for Studies of Psychological Application, South China Normal University, Guangzhou, 510631, Guangdong, China.

Xun Li, Kaizhao Chen, Yihao Long \& Xiaodong Ma

Cancer Research Institute of Zhongshan City, Zhongshan City People's Hospital, Zhongshan, 528403, Guangdong, China.

Fugui Li

\section{Contributions}

ZF, YNH and YHZ performed most experiments. LX, CK, LYY assisted in flow cytometry, Western blot analysis, and data interpretation. MX and FGL designed and wrote the manuscript. All authors reviewed the manuscript and approved the submission.

\section{Corresponding authors}

Correspondence to Fengyun Zhao or Xiaodong Ma.

\section{Conflict of interest}

All authors declared that they have no competing interests.

\section{Ethical approval}

This article does not contain any studies with human participants or animals performed by any of the authors. 


\section{Consent for publication}

All authors agreed with the content of the manuscript.

\section{References}

1. Ilic M, Ilic I (2016) Epidemiology of pancreatic cancer. World J Gastroenterol 22(44):9694-9705

2. Castaneda-Avila M, Cruz-Benitez A, Fuentes-Payan W, Maldonado-Albertorio A, Nieves-Ferrer L, PadroJuarbe $P$ et al (2016), Epidemiology of Pancreatic Cancer in Puerto Rico (1987-2010): Incidence, Mortality and Survival,Austin J Gastroenterol3 (1)

3. Goral V (2015) Pancreatic Cancer: Pathogenesis and Diagnosis. Asian Pac J Cancer Prev 16(14):5619-5624

4. Hu JX, Zhao CF, Chen WB, Liu QC, Li QW, Lin YY et al(2021), Pancreatic cancer: A review of epidemiology, trend, and risk factors,World J Gastroenterol27 (27):4298-4321

5. Parris TZ, Kovacs A, Hajizadeh S, Nemes S, Semaan M, Levin M et al (2014) Frequent MYC coamplification and DNA hypomethylation of multiple genes on $8 q$ in 8p11-p12-amplified breast carcinomas. Oncogenesis 3:e95

6. Brown DN, Caffa I, Cirmena G, Piras D, Garuti A, Gallo M et al (2016) Squalene epoxidase is a bona fide oncogene by amplification with clinical relevance in breast cancer. Sci Rep 6:19435

7. Ge H, Zhao Y, Shi X, Tan Z, Chi X, He M et al (2019) Squalene epoxidase promotes the proliferation and metastasis of lung squamous cell carcinoma cells though extracellular signal-regulated kinase signaling. Thorac Cancer 10(3):428-436

8. Liu Y, Sun W, Zhang K, Zheng H, Ma Y, Lin D et al (2007) Identification of genes differentially expressed in human primary lung squamous cell carcinoma. Lung Cancer 56(3):307-317

9. Khan NA, Stopsack KH, Allott EH, Gerke T, Giovannucci EL, Mucci LA et al(2019), Intratumoral Sterol27-Hydroxylase (CYP27A1) Expression in Relation to Cholesterol Synthesis and Vitamin D Signaling and Its Association with Lethal Prostate Cancer,Cancer Epidemiol Biomarkers Prev28 (6):1052-1058

10. Sui Z, Zhou J, Cheng Z, Lu P (2015) Squalene epoxidase (SQLE) promotes the growth and migration of the hepatocellular carcinoma cells. Tumour Biol 36(8):6173-6179

11. Liu D, Wong CC, Fu L, Chen H, Zhao L, Li C et al (2018) Squalene epoxidase drives NAFLD-induced hepatocellular carcinoma and is a pharmaceutical target,Sci Transl Med10 (437)

12. Guillaumond F, Bidaut G, Ouaissi M, Servais S, Gouirand V, Olivares $O$ et al (2015) Cholesterol uptake disruption, in association with chemotherapy, is a promising combined metabolic therapy for pancreatic adenocarcinoma. Proc Natl Acad Sci U S A 112(8):2473-2478

13. Gupta VK, Sharma NS, Kesh K, Dauer P, Nomura A, Giri B et al (2018) Metastasis and chemoresistance in CD133 expressing pancreatic cancer cells are dependent on their lipid raft integrity. Cancer Lett 439:101-112 
14. Ikonen E (2008) Cellular cholesterol trafficking and compartmentalization. Nat Rev Mol Cell Biol 9(2):125-138

15. Zhao K, Zhao Y, Zhu JY, Dong H, Cong WM, Yu Y et al (2018) A Panel of Genes Identified as Targets for 8q24.13-24.3 Gain Contributing to Unfavorable Overall Survival in Patients with Hepatocellular Carcinoma. Curr Med Sci 38(4):590-596

16. Bonne AC, den Bieman MG, Gillissen GF, van Lith HA, van Zutphen LF (2002) Chromosomal localization of genes involved in biosynthesis, metabolism or transport of cholesterol in the rat. Cytogenet Genome Res 97(3-4):183-186

17. Polycarpou-Schwarz M, Gross M, Mestdagh P, Schott J, Grund SE, Hildenbrand C et al (2018) The cancer-associated microprotein CASIMO1 controls cell proliferation and interacts with squalene epoxidase modulating lipid droplet formation. Oncogene 37(34):4750-4768

18. Qin Y, Zhang Y, Tang Q, Jin L, Chen Y (2017) SQLE induces epithelial-to-mesenchymal transition by regulating of miR-133b in esophageal squamous cell carcinoma. Acta Biochim Biophys Sin (Shanghai) 49(2):138-148

\section{Figures}


A

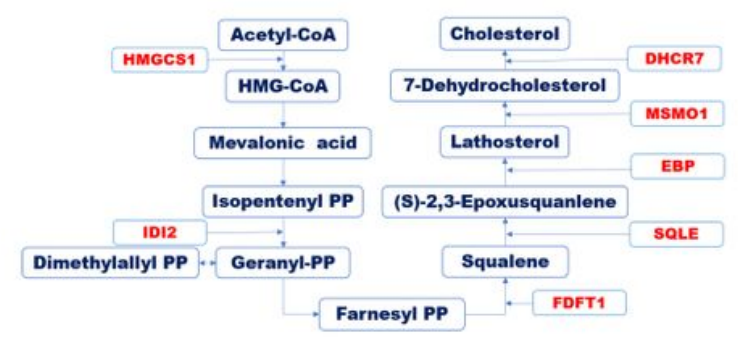

D

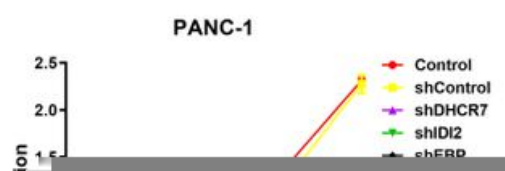

B

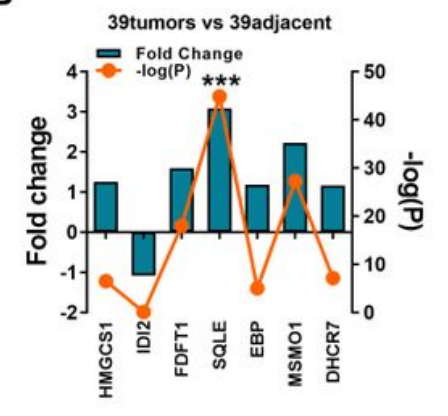

E

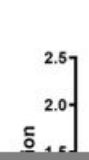

$\mathbf{F}$

BXPC3
C

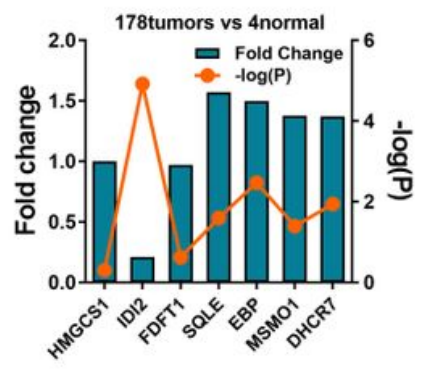

2.5
2.0
$5 . .$.
CFPAC1

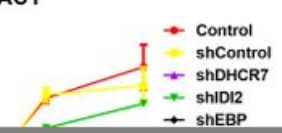

\section{Figure 1}

The role of cholesterol metabolism associated genes in cell proliferation of pancreatic cancer.

A The main cholesterol synthesis pathway. SQLE, HMGCS1, FDFT1, DHCR7, EBP, IDI2 and MSMO1 are important enzymes involved in cholesterol metabolism. B The expression level of seven cholesterol metabolism genes in 39 pancreatic tumors and its adjacent tissues from ONCOMINE database. (C) The expression level of seven cholesterol metabolism genes in 178 pancreatic tumors and 4 normal 
pancreatic tissues from TCGA database. D-F PANC-1 (D), BXPC3 (E) and CFPAC1 (F) were steady transfected with shRNA of seven genes for $24 \mathrm{~h}, 48 \mathrm{~h}$ and $72 \mathrm{~h}$ and cell viability was measured by CCK8. Data are means $\pm \mathrm{sd}$. ${ }^{*} \mathrm{P}<0.05,{ }^{\star *} \mathrm{P}<0.01$ and $* \star * \mathrm{P}<0.001$.

A

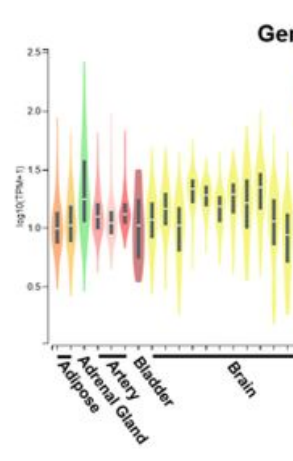

C

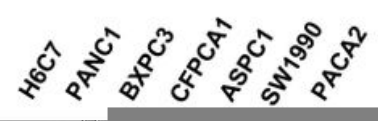

B

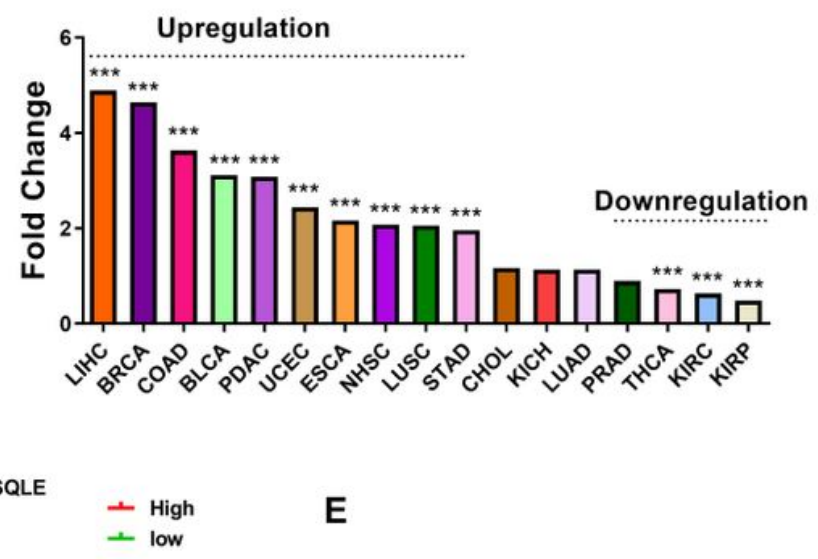

PANC-1 CFPAC1

Figure 2

SQLE is up-regulated and promotes tumor progression in PDAC . 
A The transcription levels of SQLE in human normal tissues from GTEx. B The mRNA expression of SQLE between tumor and normal samples from TCGA. C The protein expression level of SQLE in normal pancreatic cell line (H6C7) and pancreatic cancer cell lines PANC1, BXPC3, CFPAC1, ASPC1, SW1990 and PACA2. D Kaplan-Meier analysis (log-rank test) of overall survival of human pancreatic cancer samples from Kaplan-Meier Plotter database based on SQLE expression. E Knockdown of SQLE in PANC-1 and CFPAC1 cells was confirmed by Western blot. $\mathbf{F}$ The effect of knocking down SQLE on colony formation in Panc-1 and CFPAC1 cells was tested using a colony formation assay, Colonies consisting of more than 50 cells were counted by microscope with $10 \times$ objective. $\mathbf{G}$ The effect of knocking down SQLE on cell invasive ability in Panc-1 cells was tested using a transwell migration assay, Scale bars, $100 \mu \mathrm{m}$. H Cell cycle distribution was determined using propidium iodide $(\mathrm{PI})$ staining and analyzed by flow cytometry after PANC-1 cells steady transfected with shControl or shSQLE. ${ }^{\star} P<0.05,{ }^{\star \star} P<0.01,{ }^{* \star *} \mathrm{P}<$ 0.001 . 
A

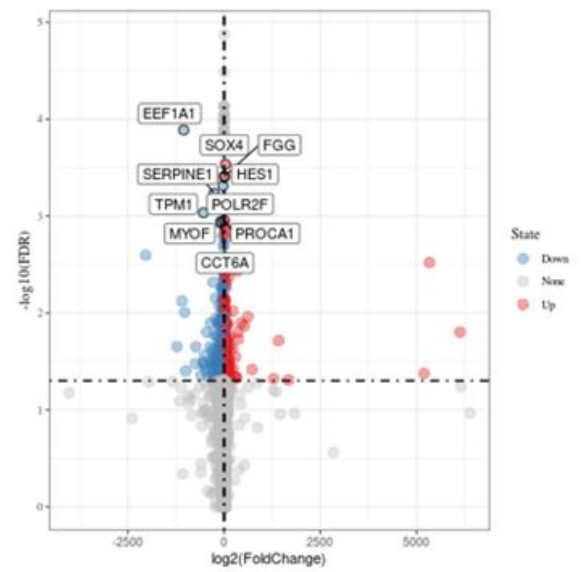

C

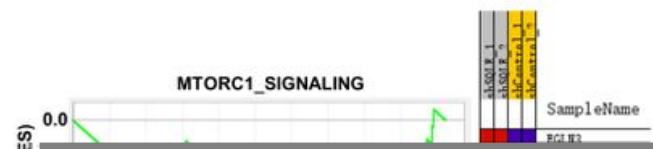

B

NES

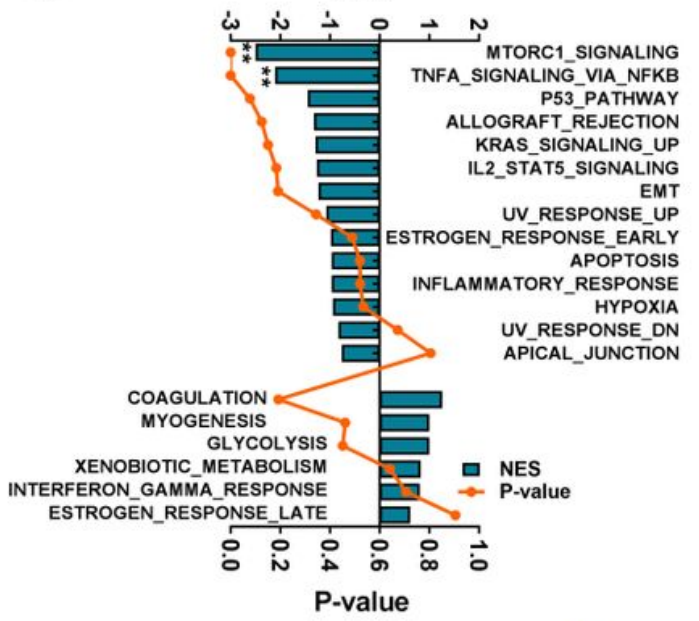

D

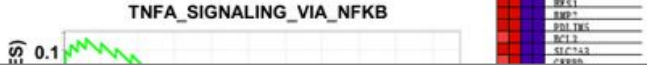

\section{Figure 3}

SQLE silencing negatively modulates mTORC1 and TNFa/NF-KB signaling in pancreatic cancer cells.

A Volcano plot shows differentially regulated gene expression from RNA-seq in PANC-1 cells steady transfected with shControl or shSQLE. B GSEA analysis of the enrichment pathways with normalized enrichment score (NES) and targets. C-D GSEA enrichment plots and heatmaps of gene expression changes in sets of mTORC1 signaling (C) and TNFa signaling via NF-KB (D). 

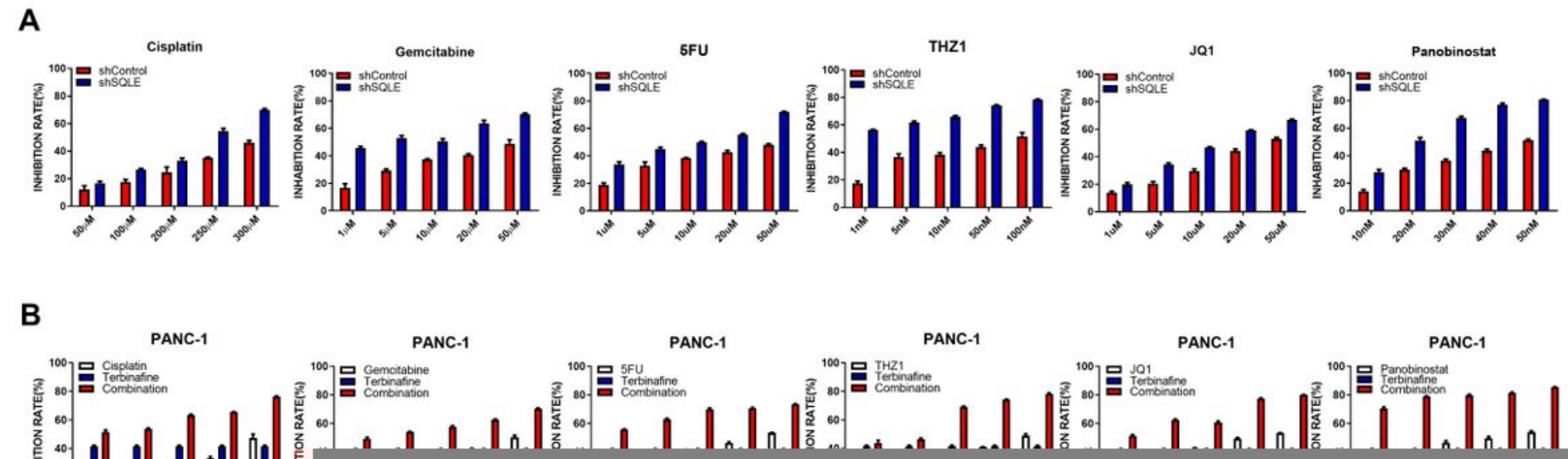

Figure 4

Terbinafine augments chemotherapeutic drug sensitivity in PDAC cell lines.

A Inhibition rate of PANC-1 steady transfected with shControl or shSQLE treated with cisplatin, gemcitabine, 5FU, THZ1, JQ1 and panobinostat at each designated concentration. B Cell viability of PANC-1 cells treated with terbinafine $(100 \mu \mathrm{M})$ and six chemotherapeutic drugs (cisplatin, gemcitabine, 5FU, THZ1, JQ1 and panobinostat) or terbinafine in combination with six chemotherapeutic drugs at each 
designated concentration (0-300 $\mu \mathrm{M})$ for $48 \mathrm{~h}$. C PDAC cell lines(CFPAC1, BXPC3, ASPC1, PACA2, SW1990) were treated with terbinafine (20-100 $\mu \mathrm{M})$, panobinostat(10-110 nM) or terbinafine in combination with panobinostat at each designated concentration for $48 \mathrm{~h}$. 